\title{
Gêneros e suas nuances no ENEM
}

\section{Viviana Elizabeth Romero Noguera ${ }^{1}$, Kalinka Regina Lucas Jaquie Castelo Branco ${ }^{1}$, Cristina Dutra de Aguiar Ciferri ${ }^{1}$}

\author{
${ }^{1}$ Instituto de Ciências Matemáticas e de Computação - Universidade de São Paulo (USP) \\ Avenida Trabalhador São-carlense, 400 - 13.566-590 - São Carlos - SP - Brasil \\ viviana.noguera@usp.br, \{cdac,kalinka\} @icmc.usp.br
}

\begin{abstract}
Aiming to investigate the gender gap in access to universities in the area of exact sciences, this paper analyzes, under several factors, the performance of the ENEM (National High School Examination) participants from 2013 to 2017. The results showed that the superiority performance of the male participants is not so evident when compared with the performance of the female participants. It is concluded that there is a need for educational public policies and more detailed analysis of a larger set of data, besides the integration of ENEM information with SISU (Unified Selection System) and ProUni (University for All Program) to help achieve the gender balance in accessing universities with a focus on exact sciences.
\end{abstract}

Resumo. Visando investigar a diferença de gêneros no acesso às Universidades na área de ciências exatas, este artigo analisa, sob diferentes fatores, o desempenho dos participantes do ENEM (Exame Nacional do Ensino Médio) nos anos de 2013 a 2017. Os resultados obtidos mostraram que a superioridade de desempenho dos participantes masculinos não é tão evidenciada quando comparada ao desempenho das participantes femininas. Conclui-se que há a necessidade de realização de políticas públicas educacionais e de análises mais pormenorizadas de um conjunto maior de dados, além da integração entre as informações do ENEM com o SISU (Sistema de Seleção Unificada) e ProUni (Programa Universidade para Todos) para auxiliar na obtenção do equilíbrio entre gêneros no acesso às Universidades com foco em ciências exatas.

\section{Introdução}

Alcançar a igualdade de gênero é um dos 17 objetivos da ONU para atingir o desenvolvimento sustentável até 2030, buscando "aumentar o uso de tecnologias de base, em particular as tecnologias de informação e comunicação, para promover o empoderamento das mulheres" [ONU 2015]. Quanto à tecnologia da informação, apesar do grande número de cursos na área de ciências exatas e do aumento de vagas para desenvolvedores e analistas de sistemas, e de profissionais formados em cursos relacionados [Thomas 2015, Statistics 2014], o número de mulheres que ingressam e se formam nessa área está decrescendo [Oliveira et al. 2014]. Até 2018, o número de mulheres ingressantes na carreira de computação é baixo e em redução a cada ano [Henn 2014, Santos 2018].

Segundo relatório da UNESCO (United Nations Educational, Scientific and Cultural Organization), adolescentes do sexo feminino não buscam as ciências exatas na mesma proporção que os do sexo masculino [UNESCO 2003]. Essa escolha reflete no 
ensino superior, havendo um desequilíbrio de gêneros nos cursos de exatas. Assim, no âmbito da educação, ações específicas precisam ser desenvolvidas para despertar o interesse das mulheres nos cursos relacionados à ciência, tecnologia, engenharia e matemática (do inglês, Science, Technology, Engineering, Mathematics, STEM). Para dar subsídios a essas ações, surgem questões importantes que, dentro do contexto deste artigo, remetem à formação das mulheres ${ }^{1}$ no ensino médio: Qual o desempenho das participantes no ENEM de sexo feminino, no âmbito das ciências exatas? e Como esse desempenho se compara ao desempenho dos participantes de sexo masculino nesse mesmo âmbito?.

O ENEM, Exame Nacional de Ensino Médio, foi criado pelo MEC para avaliar o nível de aprendizado dos alunos que concluíram o ensino médio. É o maior exame do Brasil e o segundo maior do mundo [Silveira and Mauá 2018], e contou com mais de 6,7 milhões de inscritos na edição de 2017. O exame está dividido em quatro áreas do conhecimento, Linguagens, Códigos e suas Tecnologias, Matemática e suas Tecnologias, Ciências Humanas e suas Tecnologias e Ciências da Natureza e suas Tecnologias, apresentando, ainda, eixos cognitivos comuns a todas essas áreas. Portanto, analisar os resultados do ENEM pode revelar tendências de análise interessantes no âmbito da educação.

Porém, analisar os resultados do ENEM não é uma tarefa trivial de ser realizada, devido ao grande volume de dados disponibilizados e à grande quantidade de variáveis consideradas em seus microdados. Na literatura, trabalhos voltados à análise dos resultados do ENEM (ver seção 2) são limitados, no sentido que usualmente descrevem poucos fatores de análise considerando anos específicos ou um intervalo pequeno de anos. Ademais, essas análises encontram-se defasadas, contendo resultados até 2015, no máximo.

Visando suprir essa limitação existente na literatura, e ao mesmo tempo oferecer subsídios para o desenvolvimento de ações específicas no âmbito da educação, este artigo tem como objetivo analisar o desempenho dos participantes do ENEM considerando a temática gêneros e suas nuances na tecnologia da informação. Vários fatores de análise são investigados em termos de participantes femininos e masculinos considerando as ciências exatas. Esses fatores são: região, cor/raça, tipo de escola de ensino médio e renda salarial mensal. É considerado um período recente de 5 anos, ou seja, os resultados reportados referem-se ao período de 2013 a 2017.

O artigo está organizado da seguinte forma. Na seção 2 é descrita a literatura relevante em relação ao ENEM. Na seção 3 são resumidas as características dos microdados disponibilizados pelo ENEM. Na seção 4 são ilustrados e analisados os resultados obtidos, enquanto que na seção 5 são listados desafios e direcionamentos futuros. O artigo é concluído na seção 6 .

\section{Mapeamento Sistemático}

O Mapeamento Sistemático (MS) é um estudo secundário que tem como objetivo identificar artigos relacionados a um tópico amplo de pesquisa [Kitchenham and Charters 2007]. Ele foi feito usando como suporte a ferramenta computacional StArt (State of the Art through Systematic Review) [Fabbri et al. 2012].

\footnotetext{
${ }^{1}$ As definições de sexo e gênero podem ser vistas como: o primeiro se refere às categorias inatas do ponto de vista biológico, enquanto o segundo diz respeito aos papéis sociais relacionados com a mulher e o homem, permitindo uma distinção sociológica [Moore 1997]. Entretanto, neste trabalho sexo e gênero são vistos de forma indiscriminada.
} 
O objetivo geral do MS foi identificar e classificar a literatura relevante em relação ao ENEM. Foram definidas as seguintes palavras-chave para comporem as strings de busca: Exame Nacional do Ensino Médio, ENEM e National High School Exam. Foram realizadas buscas nas bases de dados IEEE Xplore ${ }^{2}$, Scopus ${ }^{3}$ e ACM DigitalLibrary ${ }^{4}$. A fonte $\mathrm{DBLP}^{5}$ não foi considerada porque a maioria de suas publicações encontra-se disponível nas fontes mencionadas e suas capacidades de busca são limitadas quando comparadas a essas [Batista et al. 2018]. Foram considerados trabalhos publicados nos anos de 2015 até 2019, nos idiomas português e inglês.

$\mathrm{Na}$ Figura 1 é representado o processo realizado. No total, foram encontrados 117 artigos. Na primeira fase, foram excluídos 3 artigos duplicados. A avaliação de títulos e resumos resultou na exclusão de mais 39 artigos na segunda fase. Dos 75 artigos restantes, mais 33 artigos foram excluídos após a revisão dos textos completos na terceira fase. Por fim, restaram 39 artigos mais relevantes.

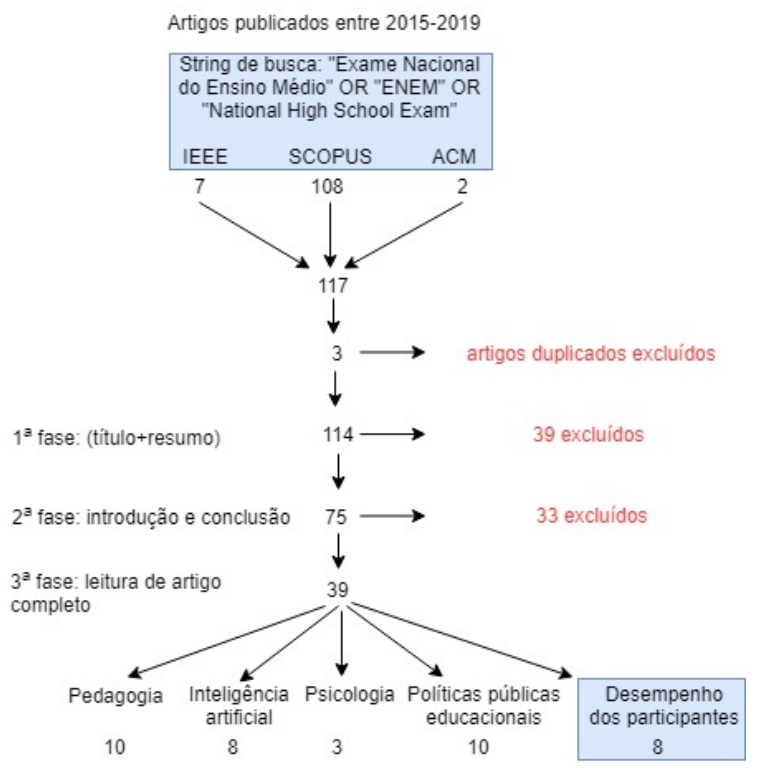

Figura 1. Processo de Mapeamento Sistemático

Os 39 artigos foram classificados em 5 categorias. A categoria pedagogia engloba 10 artigos que ajudam a melhorar a escrita da prova do ENEM e propõem políticas de accessibilidade para pessoas com deficiências. A categoria inteligência artificial inclui 8 artigos que empregam processamento de linguagem natural e aprendizado de máquina para a medição do sucesso no vestibular e a resolução de questões da prova. Na categoria de psicologia, os 3 artigos encontrados avaliam e monitoraram os padrões de sono, estresse e ansiedade dos adolescentes em relação ao vestibular, bem como analisam a euforia dos participantes do ENEM por meio de postagens no Twitter. A categoria políticas públicas educacionais engloba 10 artigos com foco nas escolas, as quais são analisadas considerando fatores como médias no exame, localização, tipo da dependência administrativa, aspectos socioeconômicos, formação do corpo docente e taxa de abandono.

\footnotetext{
${ }^{2} \mathrm{http} / / /$ ieeexplore.ieee.org

${ }^{3}$ http://scopus.org

${ }^{4} \mathrm{http}: / / \mathrm{dl}$.acm.org

${ }^{5}$ https://dblp.org
} 
Desempenho dos participantes inclui 8 trabalhos e é a categoria mais próxima ao presente artigo. Desses trabalhos, 5 investigam majoritariamente o fator socioeconômico dos participantes do ENEM e seu impacto no desempenho dos mesmos, para os anos de 2009 a 2012 [Travitzki et al. 2016], 2012 [Kleinke 2017], 2013 [Lobo et al. 2017, Da Silva et al. 2016], e 2009, 2012 e 2015 [Nascimento et al. 2018]. Um estudo do percentual de acerto nas questões da prova e a comparação desse percentual considerando o sexo, o tipo de escola e a região dos participantes para os anos de 2009 a 2013 é descrito em [Pontes Junior et al. 2016]. Já os trabalhos de [Valente 2016, Senkevics 2018] consideram os efeitos da raça, da classe e da renda no desempenho dos participantes do ENEM, para os períodos de 2004 a 2008 e de 2012 a 2015, respectivamente. Diferentemente dos trabalhos classificados nessa categoria, o presente artigo avança no estado-da-arte investigando vários fatores de análise em relação ao sexo e às ciências exatas, a saber: ano, região, cor-raça, tipo de ensino médio e renda mensal. Em especial, sexo e ciências exatas são usados para mostrar suas nuances no desempenho dos participantes no ENEM, remetendo à análise de gêneros e a tecnologia de informação. Outro diferencial é que o presente artigo analisa esses fatores considerando um período recente de 5 anos: de 2013 a 2017. Adicionalmente, o presente artigo considera, no âmbito de ciências exatas, notas gerais relacionadas às questões de ciências da natureza, matemática e linguagens e códigos, em contrapartida a trabalhos que investigam individualmente questões de física e educação física [Pontes Junior et al. 2016, Kleinke 2017, Nascimento et al. 2018]. Os fatores aqui analisados devem permitir e embasar discussões, decisões e proposições de ações que permitam reverter o quadro de desequilíbrio de gêneros nos cursos de exatas.

\section{Métodos}

Os microdados referentes ao desempenho dos participantes do ENEM são disponibilizados pelo Inep ${ }^{6}$ (Instituto Nacional de Estudos e Pesquisas Educacionais Anísio Teixeira) em arquivos no formato "csv". Existe um arquivo para cada ano de realização da prova, o qual contém microdados referentes a até 137 variáveis. Essas variáveis contêm, por exemplo, informações gerais sobre a realização das provas, a caracterização do participante e da escola que ele declarou ter frequentado, e as notas das provas objetivas e da redação. Os participantes não estão identificados pelos seus nomes. Para cada participante, é atribuído um número de inscrição, garantindo seu anonimato.

Nesse artigo, foram consideradas as variáveis relacionadas com os seguintes fatores de análise: (i) sexo; (ii) região de residência do participante; (iii) cor/raça; (iv) tipo de escola de ensino médio; (v) notas; e (vi) renda mensal. Com relação às notas, foram consideradas apenas aquelas referentes às ciências exatas, ou seja, notas das questões classificadas como ciências da natureza, matemática e linguagens e códigos. Devido ao grande volume de dados, os recortes analíticos dos arquivos foram feitos utilizando o sistema gerenciador de banco de dados Oracle ${ }^{\mathrm{TM}}$ e a linguagem de programação R.

A amostra foi constituída por todos os participantes do ENEM nos anos de 2013 a 2017 que satisfizeram aos seguintes critérios: i) ter preenchido o microdado referente ao sexo; ii) ter informado a região do município de residência; iii) ter informado a cor/raça; iv) ter preenchido a categoria de renda mensal de sua família; e v) ter participado de todas as provas da área de ciências exatas, mesmo que tenha obtido 0 (zero) em uma ou mais

\footnotetext{
${ }^{6}$ http://inep.gov.br/microdados
} 
dessas provas. Foram incluídos todos os participantes, inclusive os com necessidades especiais e os treineiros, além de todas as cores dos cadernos de provas (azul, amarelo, branco, rosa e cinza). Na Tabela 1 é detalhada a quantidade de participantes por ano da amostra, bem como a porcentagem de participantes do sexo feminino e masculino.

Tabela 1. Número de participantes do ENEM considerados nas análises.

\begin{tabular}{|c|c|c|c|}
\hline Ano & Quantidade & Feminino & Masculino \\
\hline 2013 & 5.007 .934 & $58,3 \%$ & $41,7 \%$ \\
\hline 2014 & 5.949 .253 & $57,9 \%$ & $42,1 \%$ \\
\hline 2015 & 5.604 .905 & $57,7 \%$ & $42,3 \%$ \\
\hline 2016 & 5.818 .446 & $48,0 \%$ & $42,0 \%$ \\
\hline 2017 & 4.426 .755 & $58,5 \%$ & $41,5 \%$ \\
\hline
\end{tabular}

\section{Resultados e Discussões}

A análise dos dados considerou o desempenho dos participantes, o qual foi definido como a média aritmética das notas para cada área de conhecimento das ciências exatas por sexo. Adicionalmente, o desempenho foi correlacionado a cada um dos seguintes fatores de análise: região, cor/raça, tipo de escola de ensino médio e renda salarial mensal.

De forma geral, os resultados das análises mostraram que o desempenho dos participantes masculinos foi discretamente superior ao das participantes femininas em matemática e ciências da natureza. Em linguagens e códigos, o desempenho de ambos os sexos foi quase equivalente. Os resultados detalhados são apresentados e discutidos a seguir.

Na Figura 2 é ilustrado o desempenho por região. Pode-se perceber que participantes das regiões Sudeste, Sul e Centro-Oeste, nessa ordem, obtiveram as melhores notas, independente do sexo.
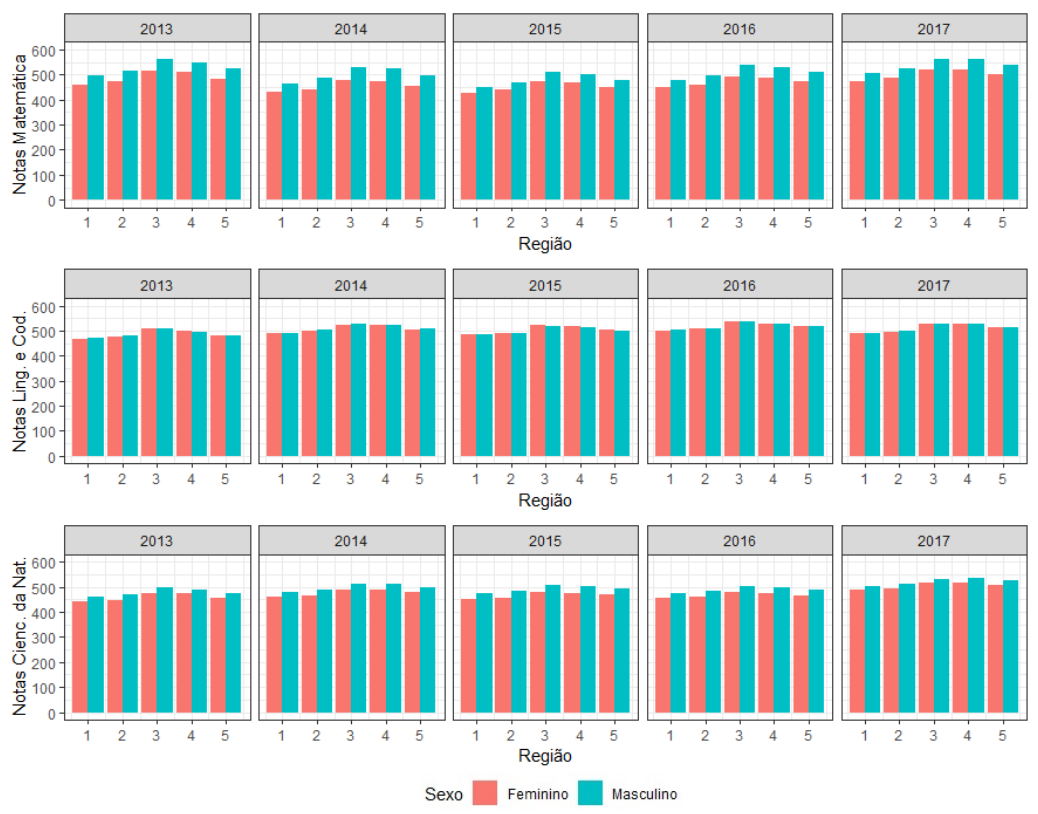

Figura 2. Desempenho por região, para as regiões identificadas como: (1) Norte, (2) Nordeste, (3) Sudeste, (4) Sul, (5) Centro-Oeste. 
O desempenho por cor/raça é mostrado na Figura 3. Nessa figura, foi usada a classificação aplicada no ENEM nos anos de 2013, 2014 e 2017. Nos anos de 2015 e 2016, os microdados também incluíram a categoria (6) não dispõe de informação. No processo de limpeza dos dados, participantes dessa categoria foram agrupados com os participantes da categoria (0) não declarado. Em todos os anos analisados, os participantes de cor/raça branca obtiveram melhores notas em matemática e ciências da natureza. Em linguagens e códigos, independente da cor/raça, as notas foram similares para todos os participantes. Por fim, participantes indígenas obtiveram as notas mais baixas das ciências exatas.
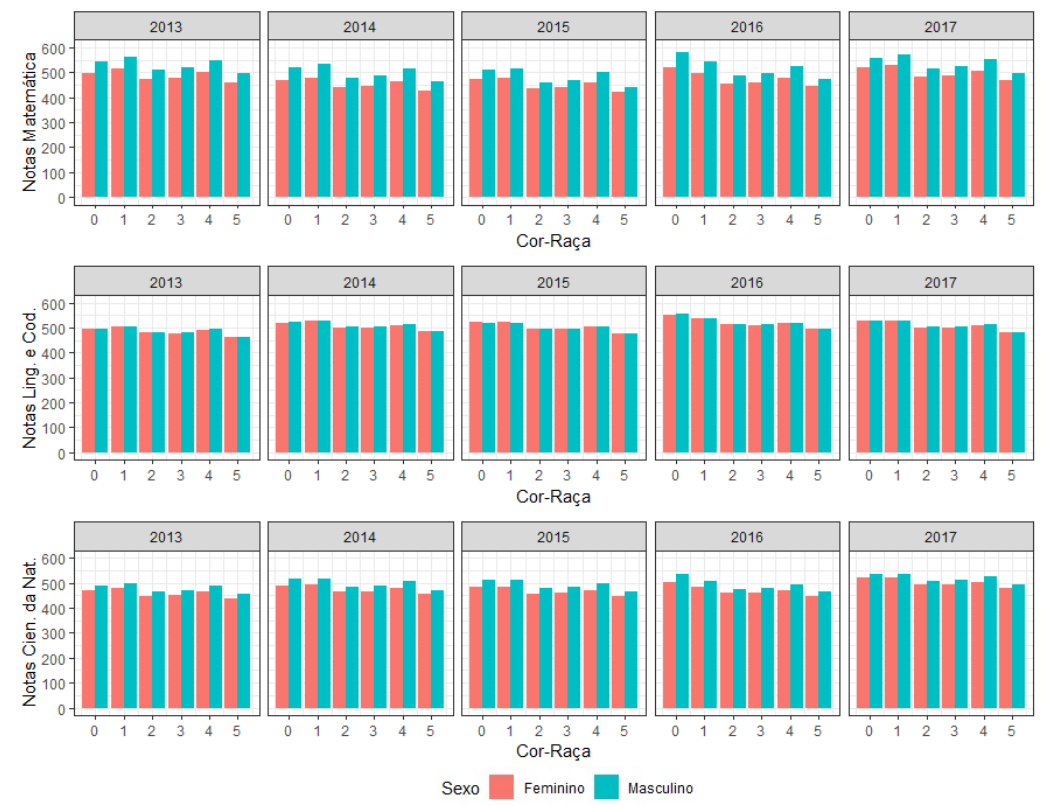

Figura 3. Desempenho por cor/raça, para as categorias identificadas como: (0) não declarado, (1) branca, (2) preta, (3) parda, (4) amarela, (5) indígena

O desempenho por tipo de escola do ensino médio é ilustrado na Figura 4. Em 2013 e 2014, os tipos de escola foram categorizados em (1) pública e (2) privada, enquanto que em 2015, 2016 e 2017 eles foram categorizados como (1) não respondeu, (2) pública, (3) privada e (4) exterior. No processo de limpeza dos dados, usou-se a categorização mais detalhada visando maior diversidade de análise. Nesse processo, participantes de 2013 e 2014 com valores nulos em tipo de escola foram reclassificados como da categoria (1) não respondeu. Para esses anos, a categoria (4) exterior não incluiu participantes por não ser possível identificá-los. Nos anos de 2013 e 2014, os resultados mostraram que os participantes oriundos de escolas privadas obtiveram melhor desempenho do que os de escolas públicas. Para os anos de 2015 a 2017, os resultados foram melhores para participantes de escolas no exterior, depois de escolas privadas e depois de escolas públicas.

Para investigar a variabilidade dos dados e valores atípicos que podem influenciar a análise dos resultados relativos à categoria (4) exterior, na Figura 5 é ilustrado o desempenho comparativo por tipo de escola do ensino médio no ano de 2017. Os resultados de 2015 e 2016 são omitidos devido às restrições de espaço. O retângulo contém 50\% da amostra. A linha interna dentro dele indica a mediana, sendo que pelo menos $50 \%$ da amostra está acima desse valor e pelo menos $50 \%$ está abaixo. Nota-se que: (i) as medianas dos participantes formados no (4) exterior são maiores do que as medianas dos 

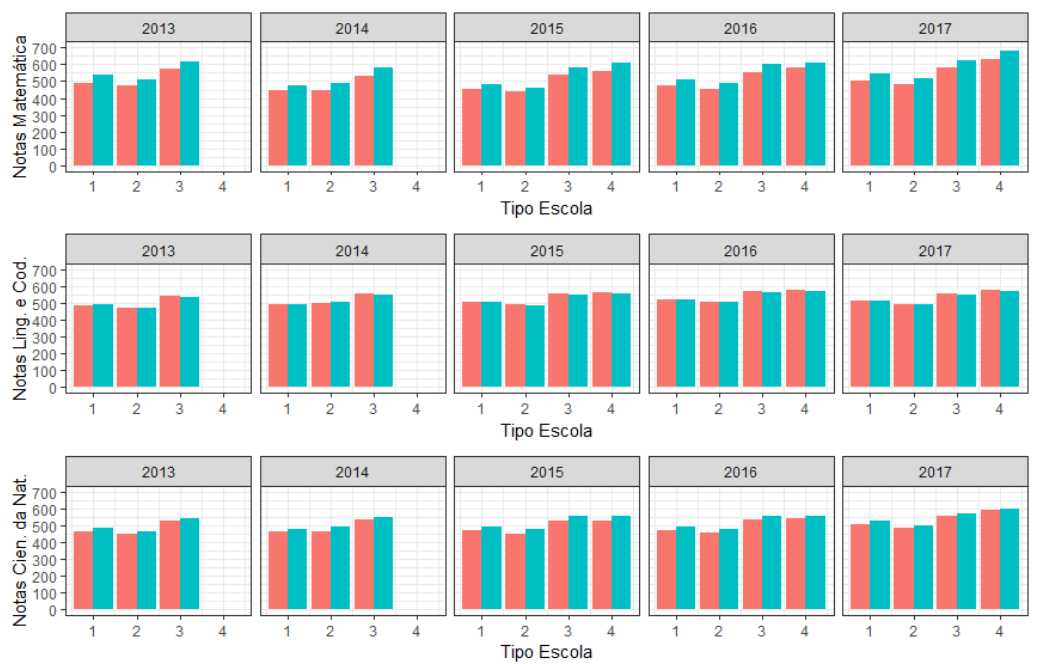

Figura 4. Desempenho por tipo de escola do ensino médio, para as categorias identificadas como: (1) não respondeu, (2) pública, (3) privada, (4) exterior.

participantes formados nos tipos de escola (3) privada e (2) pública; (ii) as notas mínimas dos formados no exterior (traço de linha horizontal mais inferior no gráfico) estão acima das notas mínimas dos formados nas escolas privadas e públicas; (iii) as notas máximas dos formados no exterior (traço de linha horizontal mais superior no gráfico) estão acima das notas máximas dos formados nas escolas privadas e públicas; e (iv) as notas mínimas mostram uma variabilidade menor de valores (pontilhados abaixo do traço horizontal mais inferior), enquanto que as notas máximas mostram uma variabilidade maior de valores (pontilhados acima do traço horizontal mais superior). A justificativa é que, comparada à quantidade de participantes das outras categorias, a categoria (4) possui o menor número de participantes: 625 em 2015, 985 em 2016 e 4.596 em 2017. Assim, à medida que a quantidade de participantes por tipo de escola aumenta, a média das notas diminui.
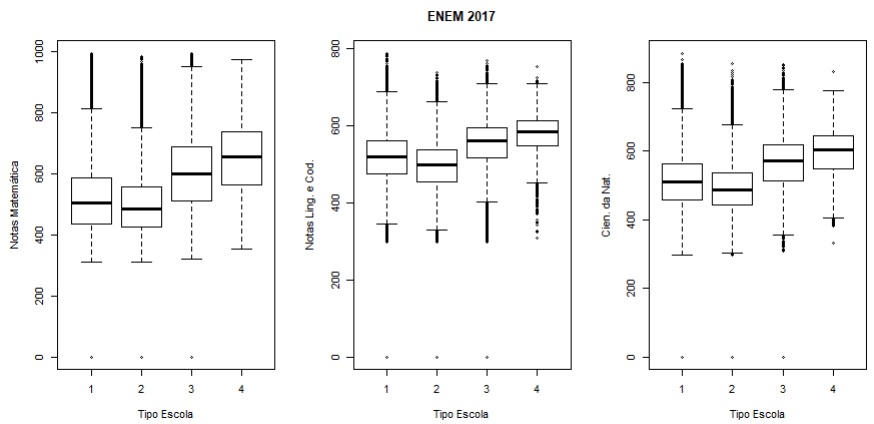

Figura 5. BoxPlot do desempenho comparativo por tipo de escola do ensino médio no ano de 2017, para as categorias identificadas como: (1) não respondeu, (2) pública, (3) privada, (4) exterior.

Na Figura 6 é ilustrado o desempenho por renda salarial mensal, considerando os valores dos salários mensais de 2017. Conforme esperado, os resultados mostraram que as diferenças socioeconômicas influenciam fortemente no desempenho dos participantes, ou seja, conforme aumenta a renda mensal, o desempenho dos participantes melhora. 

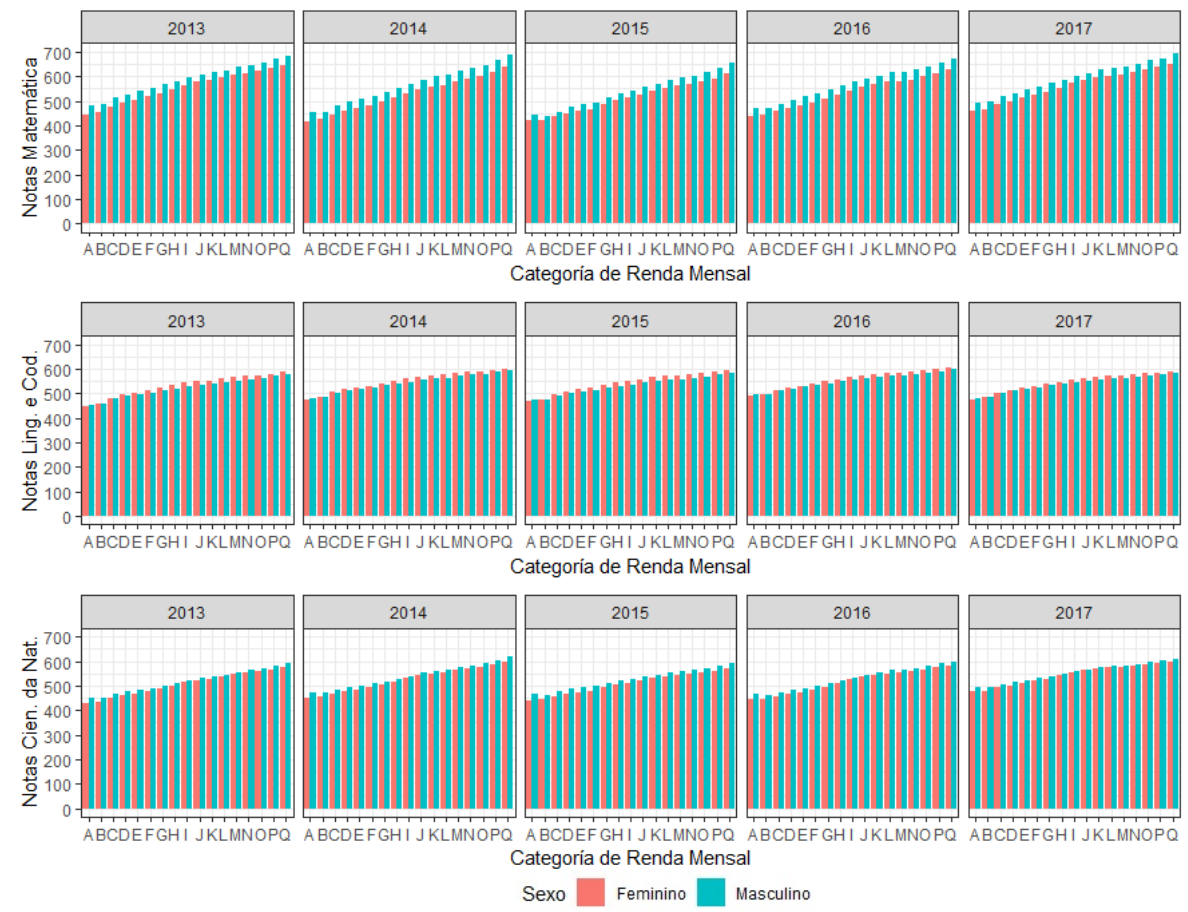

Figura 6. Desempenho por renda salarial mensal, para as categorias identificadas como: (A) nenhuma renda. (B) até $R \$ 937,00$. (C) de $R \$ 937,01$ até $R \$ 1.405,50$. (D) de $R \$ 1.405,51$ até $R \$ 1.874,00$. (E) de $R \$ 1.874,01$ até $R \$ 2.342,50$. (F) de $R \$ 2.342,51$ até $R \$ 2.811,00$. (G) de $R \$ 2.811,01$ até $R \$ 3.748,00$. (H) de $R \$ 3.748,01$ até $R \$ 4.685,00$. (I) de $R \$ 4.685,01$ até $R \$$ $5.622,00$. (J) de $R \$ 5.622,01$ até $R \$ 6.559,00$. (K) de $R \$ 6.559,01$ até $R \$$ 7.496,00. (L) de $R \$ 7.496,01$ até $R \$ 8.433,00$. (M) de $R \$ 8.433,01$ até $R \$$ $9.370,00$. (N) de $R \$ 9.370,01$ até $R \$ 11.244,00$. (O) de $R \$ 11.244,01$ até $R \$$ 14.055,00. (P) de $R \$ 14.055,01$ até $R \$ 18.740,00$. (Q) mais de $R \$ 18.740,00$.

\section{Desafios e Direcionamentos Futuros}

Vários fatores devem ser analisados na proposta de ações específicas no âmbito da educação no que tange ao equilíbrio entre gêneros nas ciências exatas. Nesse artigo, foram investigados os fatores região, cor/raça, tipo de escola de ensino médio e renda salarial mensal, considerando o sexo e as ciências exatas.

Sobre o desempenho dos participantes por nível socioeconômico, além da renda salarial mensal, é importante também investigar a classificação baseada nos indicadores do Critério de Classificação Econômica do Brasil, mantido e atualizado pela Associação Brasileira de Empresas de Pesquisa (ABEP). Ademais, no presente artigo foram analisadas notas gerais relacionadas às questões de ciências da natureza, matemática e linguagens e códigos. Outra pesquisa pertinente consiste em analisar as notas de cada questão da área de ciências exatas, o que permitirá identificar de maneira pormenorizada os fatores que influenciam a área. Com relação às amostras, foram incluídos todos os participantes. Análises mais detalhadas devem ser realizadas para se examinar o desempenho separado dos participantes com necessidades especiais e treineiros.

Outros fatores a serem considerados em pesquisas futuras também incluem investigações específicas relacionadas às escolas de ensino fundamental, uma vez que de acordo com o Relatório Global de Monitoramento da Educação (EFA Global Education 
Monitoring Report [UNESCO 2003] todos os países se comprometeram a eliminar as disparidades de gênero também na educação primária e secundária. Esses fatores incluem o desempenho dos participantes em relação à cor-raça, por região onde o participante concluiu o ensino fundamental; por tipo de dependência administrativa (federal, estadual, municipal, privada); por localização (urbana, rural) e por situação de funcionamento (escola em atividade, paralisada, extinta). Devido à importância dessas análises, elas também deveriam ser realizadas para as escolas de ensino médio. Entretanto, os microdados disponibilizados pelo ENEM não incluem essas informações para as escolas de ensino médio, sendo o tipo de escola, investigado neste artigo, o único fator disponibilizado. Pesquisas adicionais devem ser realizadas de forma a possibilitar tais análises estendidas. Adicionalmente, correlacionar ensino fundamental com ensino médio possibilitaria uma análise investigativa da vida acadêmica do participante antes de ingressar no ensino superior.

Outros direcionamentos incluem investigações para se determinar como o investimento do PIB na educação melhora o desempenho dos participantes, bem como verificar a correlação do desempenho dos participantes em relação à escolaridade dos pais.

\section{Conclusões}

Respondendo às questões levantadas na seção 1, os resultados das análises demonstraram que o desempenho dos participantes masculinos foi discretamente superior ao das participantes femininas em matemática e ciências da natureza. Já para linguagens e códigos, o desempenho de ambos os sexos foi quase equivalente.

A partir destes resultados, pode-se concluir que existe necessidade de políticas públicas educacionais e de estudos mais aprofundados que permitam a identificação dos gaps em que se deve atuar para minimizar a discrepância e o desbalanceamento entre gêneros. Pesquisas como a apresentada neste artigo podem ajudar a tomada de melhores decisões e assim lograr o progresso educacional, reduzindo as disparidades no desempenho e dessa maneira aumentando a quantidade de pessoas que acedem ao ensino superior de maneira mais balanceada quando levada em consideração a questão de gêneros.

Em pesquisas futuras serão aplicadas outras técnicas de estatística descritiva, como média, desvio padrão e variância. Trabalho de investigação científica adicional é necessário para identificar o porcentual de permanência dos alunos aprovados nas universidades a partir das notas do ENEM. Para isso, é necessário uma ligação com os dados do SISU (Sistema de Seleção Unificada) e ProUni (Programa Universidade para Todos).

Agradecimentos. As autoras agradecem às seguintes agências de fomento pelo suporte financeiro: V.E.R. Noguera - Programa Nacional de Becas de Postgrado en el Exterior Don Carlos Antonio López - BECAL; K.R.L.J. Castelo Branco e C.D.A. Ciferri - processo 442270/2018-6 CNPq; e C.D.A. Ciferri - processo 2018/22277-8 FAPESP.

\section{Referências}

Batista, N. A., Sousa, G. A., Brandão, M. A., da Silva, A. P. C., and Moro, M. M. (2018). Tie strength metrics to rank pairs of developers from github. Journal of Information and Data Management, 9(1):69-83.

Da Silva, R., Lamb, L. C., and Barbosa, M. C. (2016). Universality, correlations, and rankings in the brazilian universities national admission examinations. Physica A: Statistical Mechanics and its Applications, 457:295 - 306. 
Fabbri, S., Hernandes, E., Thommazo, A. D., Belgamo, A., Zamboni, A., and Silva, C. (2012). Managing Literature Reviews Information through Visualization. In Proceedings of the 14th ICEIS, pages 36-45.

Henn, S. (2014). When women stopped coding. NPR Planet Money, 21.

Kitchenham, B. and Charters, S. (2007). Guidelines for performing systematic literature reviews in software engineering.

Kleinke, M. (2017). Influência do status socioeconômico no desempenho dos estudantes nos itens de física do ENEM 2012. Revista Brasileira de Ensino de Física, 39.

Lobo, G. D., da Cunha Cassuce, F. C., and Cirino, J. F. (2017). Avaliação do desempenho escolar dos estudantes da região nordeste que realizaram o ENEM: uma análise com modelos hierárquicos. Revista Espacios, 6.

Moore, H. (1997). Compreendendo sexo e gênero. Companion Encyclopedia of Anthropology. London: Routledge.

Nascimento, M., Cavalcanti, C., and Ostermann, F. (2018). Uma busca por questões de física do ENEM potencialmente não reprodutoras das desigualdades socioeconômicas. Revista Brasileira de Ensino de Física, 40.

Oliveira, A. C., Moro, M. M., and Prates, R. O. (2014). Perfil feminino em computação: Análise inicial. In Proceedings of the XXXIV CSBC.

ONU (2015). Objetivo 5: Alcançar a igualdade de gênero e empoderar todas as mulheres e meninas.

Pontes Junior, J. A. F., Silva, A. G. d., Tavare, E. D., Sousa, L. A., Bastos, F. A. C., Cruz, F. N. I. d., and Almeida, L. S. (2016). Aspectos psicométricos dos itens de educação física relacionados aos conhecimentos de esporte e saúde no exame nacional do ensino médio (ENEM). Motricidade, 12:12-21.

Santos, C. M. (2018). Por que as mulheres "desapareceram” dos cursos de computação? Journal da Universidade de São Paulo.

Senkevics, A. S. (2018). Contra o silêncio racial nos dados universiários: desafios e propostas acerca da Lei de Cotas. Educação e Pesquisa, 44.

Silveira, I. C. and Mauá, D. D. (2018). Advances in automatically solving the ENEM. In Proceedings of the 7th BRACIS.

Statistics, L. (2014). US department of labor. Dostepne $w$ internecie: http://www. msha.gov.Dostę $w$ dniu, 10.

Thomas, S. J. (2015). Exploring strategies for retaining information technology professionals: A case study. PhD thesis, Walden University.

Travitzki, R., Ferrão, M. E., and Pinto Couto, A. (2016). Desigualdades educacionais e socioeconômicas na populaçao brasileira pré-universitária: Uma visão a partir da análise de dados do ENEM. Arquivos Analíticos de Políticas Educativas, 24.

UNESCO (2003). Gender and education for all: the leap to equality.

Valente, R. (2016). The vicious circle: effects of race and class on university entrance in brazil. Race Ethnicity and Education, pages 1-14. 